\title{
Comparison of Individual Classifiers and Collective Classifiers between Chinese and English
}

\author{
Yeli Shi \\ Zhejiang Ocean University, Zhoushan City, Zhejiang Province, China
}

\begin{abstract}
This paper tries to analyze the differences and similarities between Chinese individual classifiers and collective ones, between Chinese classifiers and English partitive constructions. Chinese has countless individual classifiers, most of which originated from the referent shape and forms. Both individual and collective ones have their respective principles of formation and functions, while English partitive construction "a + N1 + of + N2" equivalent to Chinese collective classifiers is far more resourceful. Both of Chinese classifiers and English partitive construction are capable of visualizing the described object and endowing it with feelings or emotions.
\end{abstract}

Index Terms - Chinese individual classifiers, Chinese collective classifiers, English partitive construction, differences and similarities

\section{INTRODUCTION}

Among the eleven word classes in Chinese, classifier is an independent category, which is not included in the ten word classes in English, for English has no such category. However, the commonality of human nature leads to the similar cognition of "quantity". Both English and Chinese have the system of classifiers, for example, yi zhang zhi (one classifier paper) in Chinese is equivalent to "a sheet of paper" in English. But the categories of quantity in English still belong to nouns (Quirk, Greenbaum, Leech \&Startvik, 1985). In Chinese language, there are more classifiers indicating individuals with strong specificity, but less classifiers indicating groups with strong universality; while in English the converse is true (Huang, 2002 \& Wang, 2001). Since Chinese has this part of speech, it is natural that Chinese classifiers are more systematic than English ones.

\section{Conception And Classification OF Chinese Classifiers And English Partitive Constructions}

In Chinese language, a word used to represent the measuring unit is called a classifier. Chinese classifiers generally can be divided into two major categories of classifiers: noun classifiers and verbal classifiers. According to semantics, noun classifiers can be divided into individual and collective classifiers, indefinite classifiers, quantitative measure words and measure words. Verbal classifiers can also be classified according to different standards. This article is only focused on individual and collective classifiers which take up the largest proportion of classifiers. In Chinese, individual classifiers should be applied in the case of measuring individual person or object. For example, yi ge ren (one classifier person "a person"), yi jian shi (one classifier event "one event"), yi tou niu (one classifier cow "one cow"), and yi shou chuan (one classifier boat "one boat"). Here ge, jian, tou, shou are individual classifiers. Collective classifiers refer to the amount exceeding more than one, which can also be called collective noun classifiers. For example, yi qun ren (one classifier people "a group of people"), yi dui mei (one classifier coal "a pile of coal"), yi zu shuju (one classifier data "a set of data"). Here qun, dui, $z u$ are collective classifiers. Collective classifiers can be further divided into quantitative one and non-quantitative ones. The frequently used quantitative collective classifiers are qun (crowd), bang (gang), huo (band), pi (batch), fu (pair), dui (couple), shuang (pair), da (dozen) and the like. By contrast, there are only two non-quantitative collective classifiers: xie (some) and dianer (a little/a few).

In English, there are no classifiers as separate parts of speech. In English, an individual person or object is signified by "a" or "an" article when the object is countable. When object is uncountable, a fixed format, "a + N1 + of + N2" is commonly used, such as "a piece of paper", "a bar of chocolate". This is also a unique way to show quantity and measure units or manner. Quirk and Greenbaum (1973) defined this structure as "partitive construction". In the case of measuring more than one objects, plural form is adopted. However, the above rules may change with the change of classifiers functions. For instance, ' $\mathrm{a}+\mathrm{N} 1+$ of $+\mathrm{N} 2$ ' can be used to represent more than a number, such as a group of people.

In the following sections, the differences between collective classifiers and individual classifiers in Chinese, and the differences and similarities of classifiers between English and Chinese will be illustrated in details.

\section{The Basic Differences Between Collective Classifiers And Individual Classifiers in Chinese}


In meaning, collective classifiers indicate an amount of more than one, while individual classifiers only a single one.

In role, collective classifiers emphasize number, while individual classifiers category. $\operatorname{Li}(2000)$ holds the view that some of group classifiers (namely collective classifiers), such as "flock, crowd, gang, and group", mean "a number of", for example, "a number of sheep" can be collectively called "a flock of sheep" and "a number of people" "a gang/group of people". In fact, almost all collective classifiers express a certain number, provided that the noun it modifies is discrete. Besides some collective classifiers which indicate fixed numbers like "double, pair" which equal two, "dozen" which equals twelve, "ream" which equals 500, and "a gross" equals 12 dozens, there are other ones, which in a given context, also indicate definite numbers or relatively definite number, say "batch", one batch may be 20 persons, or "flock", one flock may contain 30 or so. Individual classifiers have the prominent classifying function in the examples of yi zhang zhuozi (one classifier table "one table"). The classifier zhang stresses the dimensions of a table, indicating it is a relatively large plane object. Whereas, in yi pai zuozi ("a row of tables"), pai is a collective classifier which focuses on the amount, indicating there are many tables. The number of "one row", arranged in line, in a certain context, is a fixed number.

In combination, there are four major differences between collective and individual classifiers:

Firstly, in the combination of "numeral $+X+N P$ ", if X is a collective classifier, when X is removed, the format turn s into "number + NP", which is not only grammatically inappropriate, but also semantically different from the original meaning; if X is an individual classifier, when X is removed, the format turns into "numeral + NP", which is only grammatically inappropriate, but semantically unaffected. For instance, if the classifier tou is removed from yi tou zhu (one classifier pig "one pig"), yi zhu (one pig) is still understandable and unaffected in meaning, though syntaxically wrong. In contrast, in the case of yi qun zhu (one classifier pig "a herd of pigs"), qun is a collective classifier. If it is removed, yi qun zhu turns into yi zhu, thus the meaning is utterly different in addition to the wrong syntax. Therefore, if there is a classifier omitted in a given context, the omitted one must be an individual classifier.

Secondly, in the "numeral + X" combination, if X is a collective classifier, the combination can often be inserted such adjectives as "big, small, long, thin and thick"; if X is an individual classifier (excluding those classifiers which describe the shape like "particle, slice, strip, block"), the combination, in most cases, cannot be inserted any other elements. For example, in yi zhi wuya (one classifier crow "one crow"), or liang shan chuanghu (two classifier windows "two windows"), any other word such as $d a$ (big) or xiao (small) cannot be added before the individual classifier zhi or shan. Whereas, some adjective can be inserted between numeral and collective classifier: $d a$ (large) can be added after numeral in the example of yi pi huowu (one classifier goods "a batch of goods"), or xiao (small) before the collective classifier guo in san guo jiaozi(three classifier dumplings "three pots of dumplings"). In brief, in most cases, collective classifiers can be inserted adjectives while individual classifiers cannot.

Thirdly, in the combination of "numeral + X + NP", if NP is a collective noun, X must be a collective classifier; if NP is an individual noun, $\mathrm{X}$ must be an individual classifier. For instance, yi pi qiangzhi (one classifier guns "a batch of guns"), qiangzhi (guns) is a collective noun, so pi (batch) is a collective classifier. In the example of yi zhi budui (one classifier army "one army"), army is a collective word, so zhi is a collective classifier which indicates a group of people who work together. By contrast, some objects are commonly regarded as single one, in that case, collective classifiers cannot be used to describe them. For example, yi ge diqiu (one classifier earth "one earth") cannot be said into yi qun diqiu (one classifier earth "a group of earths"), for qun is a collective classifier. But in some cases, it is hard to judge whether NP in a combination is an individual or a collective noun such as "student, mobile phone, book", then X becomes a critical element which determines NP's attribute. If $X$ is an individual classifier, then NP is a separate individual; if $\mathrm{X}$ is a collective classifier, then NP is a collection of things or the whole.

Lastly, if classifier $\mathrm{X}$ is combined with Chinese character dan (single), i.e. dan $+\mathrm{X}$, then the classifier must be an individual one. While in the formula of cheng (in considerable numbers or amounts)+ $\mathrm{X}$, the classifier is a collective one. For instance, dan ge (single one), dan zhang (one piece), dan zhi(single one), ge, zhang, zhi are all individual classifiers; cheng shuang( in pairs), cheng $z u$ (in group), cheng lei ( in category), here shuang, $z u$, and lei are collective classifiers. Commonly, dan $+\mathrm{X}$ sets "single $\mathrm{X}$ " in the background of the whole so as to highlight single itself. By contrast, cheng $+\mathrm{X}$ takes the whole as its value orientation. For instance, cheng tao (a complete set) sells at 100 yuan, dan ge (a single one) at 12 yuan $\mathrm{A}$, here cheng is the value orientation of the whole, whereas, dan ge is to show itself with the whole as its background.

Whether English has classifiers or not has been disputed by scholars. The common consensus is that English has functional partitive constructions similar to Chinese classifiers, though it has no classifiers. There exist some differences and similarities between the two. English partitive constructions and Chinese classifiers are, in essence, have very similar functions in spite of their differences in expression. They are both aimed at satisfying the need of human cognition. And the choice of the classifier is decided by the change of cognitive subjects or cognitive perspectives.

\section{The Differences Between Chinese Classifiers AND English PARTITIVE Constructions}

\section{A. Individual Classifiers}

From the perspective of signifying individual things, Chinese language, compared with English language has much richer classifiers which has formed a separate category. The fact that English uses a/an article to signify countable individuals also determines the richness of Chinese individual classifiers, which also reflects people's understanding of 
the same thing from different cognitive angles. "One object for multiple classifiers" is the result of the switch of perspectives of cognitive subjects' (Wang, 2007). If the perspectives are different, the objects' salient properties are different and the classifiers chosen change accordingly. As Wang (2001) states in his book using different classifiers on one thing may show different emotions of the language user.

For instance, the description of the moon in Chinese, yi ya/wan xinyue (one classifier crescent moon "the crescent moon"), yi gou/hen canyue (one classifier waning moon "the waning moon"), yi yuan haoyue (one classifier bright moon "the bright moon"), yi lun yиanyue (one classifier full moon "the full moon"). Different classifiers leave people with varied imagination. As the above examples illustrate, ya (tooth) is the most intuitive classifier to describe the crescent moon, which resembles the shape of a tooth, long and lathy, with slight curves at both ends. The classifier wan (curve), meaning "not straight, or bent", is commonly used in Chinese to describe the new moon with the feminine beauty. The original meaning of gou (hook) is a tool which hooks up things, with bent shape and sharp ends, highlighting the fineness of the waning moon. The classifier hen (trace) is an indication that something has been present, a mark left by objects such as lei hen (tearstain), shang hen (scar), often implying something is gone with kind of helpless feelings. hen used to describe the moon is also aimed at highlighting the feeling of loss and helplessness. Yuan (circle) is a classifier to describe the shape of the moon-- round, that is, every part of the line is the same distance from the center of the area, which presents a perceptual intuition. The classifier lun (wheel) literally referring to a car wheel, or a carriage wheel also shows a circular shape which is an appropriate description of the full moon. These examples indicate that a Chinese individual classifier can describe the shape of the object in addition to its quantitive function. Besides, it also has the function of expressing one's emotions and feelings, which is illustrated by the following two examples. One is the description of a fish, such individual classifiers as tiao ( strip), wei (tail), tou (head) can be used. tiao refers to a long and narrow thing. It is most frequently used to describe a fish, for it can present the image of the fish who swims freely with its smooth body. wei originally means the projected end of birds, beasts, insects, fish and so on. The use of wei to describe a fish is to stress the tail of the fish resembles that of an animal, long and thin. tou is the upper part of the human body or the front part of the body in animals. The use of tou can lead people's attention to the head of the fish, often used in describing a big fish like whales, sharks and other large fish.

The other example is the description of a wall. Such individual classifiers as dao, mian, and $d u$ are frequently used to modify a wall in Chinese. dao means "road" which is long and straight, and is often used to modify long-shaped objects like river and thread. Here dao is used to depict the long and towering wall. mian originally refers to "face"with the extended meaning of " flat surface", used in describing a short and broad wall. $d u$ has the meaning of fending or blocking, displaying the wall is tall and broad and hard to cross. In ancient time, noble or rich families had tall walls surrounding the courtyard which implied that poor families were denied to access. If a girl was married to such families and found that the arranged marriage unhappy, then the marriage became yi du gao qiang (one classifier tall wall "one tall wall"), which could not be crossed and reflected the helplessness of the girl who had to accept her miserable fate. There are more examples of such descriptive individual classifiers in Chinese. Therefore, it can be concluded that Chinese classifiers not only function as measuring tools, but also serve as auxiliary adjectives to express people's feeling and emotion by modifying individual objects. It is not uncommon to find a particular noun co-occurring with different classifiers to highlight different aspects of its meaning (Becker, 1975, p.113).

\section{B. Collective Classifiers}

From the perspective of quantifying collective things, English has more quantitative collective classifiers than Chinese. In Chinese, the commonly used quantitative collective classifiers are qun (crowd), bang (gang), huo (band), pi (batch), fu (pair), dui (couple), shuang (pair), da (dozen) and the like, as mentioned above. Though these collective classifiers like qun (group), bang (gang), huo (band), pi (batch) can all mean "a group of", they are different in numbers and emotional connotations. qun, bang and huo can be used to describe a group of people, in which, qun is relatively neutral, bang and huo are labeled with derogatory meaning. bang contains more people than huo. It is evident that Chinese language has abundant collective classifiers, but it is still incomparable to such structures in English.

Take qun for example, in Chinese, in the formula of yi(one) + qun(crowd/group)) + persons, the counterparts of the collective classifier qun are varied in English: "a circle of friends, a company of students, a crowd of children, a group of people, a galaxy of scholars, a throng of pedestrians, a flock of sheep, a pack of wolves, a swarm of bees, and a crash of rhinoceroses". Here "circle, company, crowd, group, galaxy, throng, flock, pack, swarm and crash" are all translated into qun in Chinese. Naturally, the use of different collective classifiers leads to different connotations and feelings. For instance, in "a crowd of children", "crowd" enables people to form a picture of a large number of noisy children; in "a galaxy of scholars", galaxy (any of the large groups of stars which make up the universe) presents a vivid scenario of many scholars gathering together like stars because of their academic achievements; in "a crash of rhinoceroses", "crash" (the noise made by a violent fall, blow the or breaking) modifies the rhino, depicting the fierceness of a great number of huge fast-moving animals, making tremendous noise and kicking up dust while treading heavily on the ground.

In addition, a fresh way of expression which has the similar structure as the above ones occurs in English language such as "a blunder of boys", "a giggle of girls", "an eloquence of lawyers", and "a skulk of thieves". The original meaning of "blunder" is a stupid unnecessary mistake. With "blunder" as a modifier, the boys' carelessness and their liability to make stupid mistakes are fully displayed. "giggle" means "to laugh quietly in a silly childish, uncontrolled 
way". Such description of the girls forms a vivid picture that the girls cannot help laughing in a childish but adorable way. "eloquence" means the ability to express ideas and opinions readily and well, so that the hearers are influenced. When it is used to modify a lawyer, the lawyers' bearing during an eloquent argument and their dashing along their speeches are dynamically shown. "Skulk" means to hide or move about secretly, trying not to be noticed, usually through fear or for some bad purpose, which exposes the thief's stealthiness incisively. The application of this structure increasingly appears in English language, that is, N1 is substituted by a meaningful noun or verb to modify N2, aimed at reflecting the state of N2. In this construction, N1 cannot be simply translated into Chinese "qun". Instead, some appropriate adjective must be added before N2 to present the vivid image of the source text.

\section{The Causes of the Differences between Chinese Classifiers and English Partitive Constructions}

Chinese language has more individual classifiers and less collective ones than English language. The difference does not exist without reason. English has countable and uncountable nouns. The singular number of individual countable nouns can be signified by a/an article, while in Chinese, the singular one number should be paired with a classifier to signify an individual object. For instance, the counterpart of "a flag" in English is yi mian qizhi (one classifier flag "one flag") in Chinese. mian, a classifier must be present between "one" and "flag" to express a flag, which makes Chinese focus more on the use of classifiers, paying attention to every nuance of individual classifiers. And in English, since there exists such structure as "a + N1 + of + N2", which can generate many variants because of its flexibility, e.g. a loaf of bread, a piece of bread or a slice of bread; a group of people, a crowd of people and so on.

\section{Similarities Between Chinese Classifiers And English PARTitive Constructions}

Although Chinese classifiers and English partitive constructions are quite different in form, they have many in common in the aspect of their functions. They can be used not only as a measure unit but also as a connotative word. The use of classifiers itself is to enrich language and one of the most important functions is to give life to language.

\section{A. Visualization of Concrete Objects}

Both Chinese classifiers and English partitive constructions can visualize the modified objects. Take five expressions for example, in Chinese: (1) yi ye bian zhou (one classifier small boat "one small boat"), ye is an individual classifier; (2) yi dai yuan shan ( one classifier distant hills "distant hills"), dai is a collective classifier; in English: (3) a blade of grass (partitive construction to describe the individual thing); (4) a mountain of newspapers (partitive construction to signify countable collective thing); (5) a blanket of snow (partitive construction to describe uncountable collective thing).

The first example using a leaf as the classifier of the boat to compare a boat on the lake or river to a willow leaf showing vividly the shape of a boat on a broad surface of the lake. The second one uses dai (belt/strip), whose original meaning is a belt made of fur, fabric or yarn. The classifier can embody the long stretch of distant hills; "blade" in the third one, originally used to describe blade of knives, swords and chisels, gives a "flat, long and narrow" feeling of a grass. In the fourth one, "mountain" which refers to the "mass of very high land going up to the peak" is often used to describe high, towering objects. Here it describes newspapers heaped together like a mountain; "blanket" in the fifth one which means "thick, woolen covering on beds" is always associated with the thick, soft, comfortable feeling, which endows snow with such feature.

\section{B. Visualization of Abstract or Conceptualized Objects}

Chinese classifiers and English partitive constructions are capable of visualizing abstract or conceptualized objects as well as specific objects, making abstract objects more visible and perceivable, and creating a profound, novel and artistic conception. For example, yi xian xiwang (one classifier hope "a ray of hope"), xian is a classifier meaning "a thread, a long and thin piece of a material such as cotton, nylon, or silk, especially one that is used in sewing". Using xian to modify "hope" highlights the hope is slim, which is more imaginative than the direct expression of "a slim hope"; yi jian buxing (one classifier misfortune "a shoulder of /considerable misfortune"), here jian functions as a classifier. The expression appears in such a sentence as: the widow shouldered yi jian buxing and set off to a life journey full of uncertainty. Using shoulder to modify misfortune makes the invisible misfortune visible or intangible tangible and turns an abstract thing into a concrete one, which enables the reader to feel the heavy burden borne by the widow.

In English, "a little breath of spring", the original meaning of "breath" is "a single act of taking in and sending air out". Here, "breath" seems to send out a fresh and sweet fragrance of the spring, which can be smelt and tasted and thus makes intangible air visible and perceivable; "a world of differences", "world" can mean everything that exists anywhere. It can refer to the earth or the planet, suggesting vastness and boundlessness. Consequently, the expression "a world of differences" implies great disparities.

\section{The Basic Equivalence of Chinese Non-quantitative Classifiers and English Ones}

Just as above mentioned, there are many quantitative classifiers in both languages. They have similarities and differences. However, regarding non-quantitative classifiers, they are nearly equivalent. In Chinese, the commonly used non-quantitative classifiers are xie (some) and dian'er (a little). xie is more than dian'er. In English, those non-quantitive expressions are often used, including "lot, quantity, deal, number, plenty", they have nuances in usage though. The following expressions are arranged in proper order of their quantity: a few (not many), a lot of (not in large 
quantity), plenty of (enough), a large number of/a large quantity of (considerable amount, the former for countable nouns, the latter for an uncountable noun). But from the cognitive perspective, the quantity standard varies with different people, that is, each person has his own view of whether the amount is small or large. The same amount may be small for one person but large for another person. For example, a very poor man who gets a higher pay than usual, will think that he has "a large quantity of money", while that money is only a small sum of money for a wealthy man. In view of each one's cognitive differences in non-quantitative structure, English and Chinese non-quantitative classifiers are basically equivalent in that "a few" is often translated into dian'er, "a lot of, plenty of, a large number of, a large quantity of" can either be translated xie or henduo (many/much) in Chinese.

\section{The Causes of the Similarities between Chinese Classifiers and English Partitive Constructions}

Considering the similarities between Chinese classifiers and English partitive construction, it is necessary to mention the function of classifiers as a unit of measurement, measuring objective things. Moreover, it is evident from the above analysis, classifiers also have descriptive function to picture the shape or appearance of objective things. Just as Foley(2001, P. 233) states classifier semantics may appeal to perpetually salient features of the typical referents of the nouns they classify. Their primary function is to provide sufficient descriptive information for the communicative purposes of human speakers in ongoing social discourse. The use of the classifier is, to a great degree, affected by the way the cognitive subject perceives the object. For instance, whether to use the Chinese classifier dao or mian or $d u$ in describing a wall is determined by the perceiver or the cognitive subject. In description of the children, whether to use "a group of children", or "a crowd of children", is also decided by the cognitive subject. As to using the same classifier on different nouns, there also exist many examples. For instance, tiao is the right classifier for "a rainbow", "a leg", "a snake", "a cucumber" and "a scarf", for tiao reflects the common longitudinal property of these objects, which is perceptually salient, despite that the intrinsic properties of the five objects vary (Ni, Gao \& Ouyang, 2009). Therefore, the internal demand is the same of Chinese classifiers and English partitive constructions. The use of different classifiers on the same object or the same classifier on different objects is based on the cognitive perspective of the perceiver. It varies with the change of the cognitive perspectives.

\section{CONCLUSION}

This paper analyzed the differences and similarities between Chinese individual classifiers and collective ones, between Chinese classifiers and English partitive constructions. The following conclusions can be drawn positively based on the above study. Firstly, Chinese has countless individual classifiers whose origins are very imaginative and life-like. The origin of most Chinese classifiers often comes from the referent shape and forms. Individual classifiers and collective ones have their respective principles of formation and functions. Secondly, Chinese has richer individual classifiers than English while English has far more collective classifiers than Chinese. Thirdly, the use of Chinese classifiers or English partitive constructions can visualize abstract and conceptualized things, enabling the described object to be more vivid and life-like. Lastly, though Chinese classifiers and English partitive constructions differ in form, they have the fundamental commonality in that the selection of Chinese classifiers or English partitive constructions is decided by the cognitive perspective of the cognitive subject.

\section{REFERENCES}

[1] Becker, A.L. (1975). A linguistic image of nature: The Burmese numerative classifier system. Linguistics, 165, 109-121.

[2] Foley, W.A. (2001). Anthropological linguistics:An introduction. Beijing: Foreign Language Teaching and Research Press \& Oxford: Blackwell Publishers Ltd.

[3] Huang, Y.J. (2002). Comparison between measure words in Chinese and English. Journal of Huainan Vocational and Technical College, 4, 84-87.

[4] Li, Y.M. (2000). The Kink of Measure words, Numerals and Nouns. Language Teaching and Linguistic Studdies, 3, 50-58.

[5] Ni, W., H.H. Gao \& Sh. X. Ouyang. (2009). Application of cognitive strategies to Chinese noun classifier E-learning. Proceedings of the 8th International Conference on Computational Semantics, 1, 305-309.

[6] Quirk, R. \& S. Greenbaum. (1973). A university grammar of English. London: Longman Group, Ltd.

[7] Quirk, R., S. Greenbaum, G. Leech \& J.Startvik. (1985). A Comprehensive grammar of the English language. London: Longman Group, Ltd.

[8] Wang, F. X. (2001). The contrastive semantics. Beijing: Foreign Language Press.

[9] Wang, X.L. (2001). Comparison between Chinese and English quantifiers. Journal of Nanjing University of Aeronautics and Astronautics (Social Science), 1, 42-44.

[10] Wang, W.B. (2007). Interpretation of cognitive construction of metaphor. Shanghai: Shanghai Foreign Language Education Press.

Yeli Shi was born in Zhejiang, China in 1967. She received her M.A. degree in English Language and Literature from Zhejiang University, China in 2004.

She is currently an associate professor in the School of Foreign Languages, Zhejiang Ocean University, China. Her research interests include cognitive linguistics and teaching methodology. 